ISSN 0103-5150

Fisioter. Mov., Curitiba, v. 26, n. 2, p. 369-377, abr./jun. 2013

Licenciado sob uma Licença Creative Commons

\title{
Variáveis bioquímicas, antropométricas e pressóricas como indicadores de risco cardiovascular em servidores públicos
}

\author{
Biochemical, anthropometric and blood pressure variables \\ as indicators of cardiovascular risk on public employees
}

\author{
Renata Aparecida Rodrigues de Oliveira ${ }^{[a]}$, Osvaldo Costa Moreira ${ }^{[b]}$, \\ Priscila Rita Niquini Ribeiro Lopes ${ }^{[\mathrm{c}]}$, William Amorim ${ }^{[\mathrm{d}]}$, Michele Silveira Breguez ${ }^{[\mathrm{e}]}$, \\ João Carlos Bouzas Marins ${ }^{[\mathrm{f}]}$ \\ [a] Mestranda em Educação Física pela Universidade Federal de Viçosa (UFV), Viçosa, MG - Brasil, e-mail: \\ renata.oliveira@ufv.br \\ [b] Professor da Universidade Federal de Viçosa (UFV), Florestal, MG - Brasil, e-mail: moreiraoc@yahoo.com.br \\ [c] Graduação em Educação Física pela Universidade Federal de Viçosa (UFV), Viçosa, MG - Brasil, e-mail: \\ pniquiniufv@yahoo.com.br \\ [d] Graduação em Educação Física pela Universidade Federal de Viçosa (UFV), Viçosa, MG - Brasil, e-mail: \\ amorimwa@yahoo.com.br \\ [e] Graduação em Educação Física pela Universidade Federal de Viçosa (UFV), Viçosa, MG - Brasil, e-mail: \\ msbreguez@yahoo.com.br \\ [f] Professor da Universidade Federal de Viçosa (UFV), Viçosa, MG - Brasil, e-mail: jcbouzas@ufv.br
}

\section{Resumo}

Introdução: As doenças cardiovasculares têm sido apontadas como a principal causa de mortalidade e morbidade no Brasil e no mundo. Objetivo: Verificar a prevalência dos fatores de risco cardiovascular em servidores universitários, utilizando as variáveis bioquímicas, antropométricas e pressóricas. Materiais e métodos: Participaram do estudo 107 servidores técnico-administrativos da Universidade Federal de Viçosa, no ano de 2010, com idade média de 46,1 \pm 10,4 anos. Os parâmetros analisados foram: índice de massa corporal, relação cintura-quadril (RCQ), pressão arterial sistólica (PAS), pressão arterial diastólica (PAD), colesterol total (CT), triglicerídeos (TG), lipoproteína de alta densidade (HDL-C) e lipoproteína de baixa densidade (LDL-C). A análise dos dados consistiu no cálculo das prevalências das variáveis estudadas. Para todos os tratamentos estatísticos adotou-se um nível de significância de $\mathrm{p}<0,05$. Resultados: Foram 
encontrados $45 \%$ de excesso de peso (IMC $\geq 25,0 \mathrm{~kg} / \mathrm{m}^{2}$ ), $20 \%$ da população apresentou RCQ elevada $(>0,85$ para as mulheres $\mathrm{e} \geq 0,95$ para os homens), a pressão arterial se apresentou alta (PAS $\geq 140 \mathrm{mmHg}$; $\mathrm{PAD} \geq 90 \mathrm{mmHg}$ ) em $24 \%$ dos avaliados, o CT obteve $49 \%$ de valores elevados (> $199 \mathrm{mg} / \mathrm{dL}$ ), os triglicerídeos apresentaram $23 \%$ valores alterados (> $149 \mathrm{mg} / \mathrm{dL}$ ), em relação ao LDL-C foram encontrados $31 \%$ de valores acima de $129 \mathrm{mg} / \mathrm{dL}$, e $30 \%$ dos avaliados apresentaram valores reduzidos de HDL-C ( $<40 \mathrm{mg} / \mathrm{dL}$ ). Observamos que apenas $18,6 \%$ dos avaliados não apresentaram nenhum dos FRC analisados, sendo que cerca de metade dos servidores se encontraram na faixa de 2 a 4 FRC. Conclusão: Foi verificado um elevado percentual de FRC na população de servidores universitários. Dentre as variáveis analisadas, verificamos que excesso de peso, CT, níveis reduzidos de HDL-C e aumentados de LDL-C foram os FRC mais prevalentes.

Palavras-chave: Epidemiologia. Saúde pública. Doenças crônicas. Sistema cardiovascular.

\section{Abstract}

Introduction: Cardiovascular diseases have been identified as the main cause of morbidity and mortality in Brazil and in the world. Objective: To verify the prevalence of cardiovascular factors risk (CFR) over university employees, through the use of biochemical, anthropometric and blood pressure variables. Materials and methods: Participated of the study 107 technical-administrative employees of Universidade Federal de Viçosa, in 2010, with the average age of 46,1 $\pm 10,4$ years old. The analyzed parameters ware: level of body mass (BLM), waist-hip ratio (WHR), systolic arterial pressure (SAP), diastolic arterial pressure (DAP), total cholesterol (TC), triglycerides (TG), high-density lipoprotein (HDL-C) and low-density lipoprotein (LDL-C). The data analysis was to calculate the prevalence of the variables studied. For all statistical treatments was adopted $p<0.05$ as significance level. Results: It was found $45 \%$ of over-weight (BLM $\geq 25.0 \mathrm{~kg} / \mathrm{m} 2), 20 \%$ of population showed higher WHR ( $\geq 0.85$ for women and $\geq 0.95$ for men), the blood pressure was also high (SAP $\geq 140$ $\mathrm{mmHg}$; DAP $\geq 90 \mathrm{mmHg}$ ) in $24 \%$ of evaluated, the TC obtained $49 \%$ of high values (>199 $\mathrm{mg} / \mathrm{dL}$ ), triglycerides showed $23 \%$ of changed values (> $149 \mathrm{mg} / \mathrm{dL}$ ), although the $\mathrm{LDL}-\mathrm{C}$ were found $31 \%$ of values over $129 \mathrm{mg} /$ $d L$, and $30 \%$ of evaluated showed low values of $H D L-C(<40 \mathrm{mg} / \mathrm{dL})$. It was possible to observe that just $18.6 \%$ evaluated did not show any CFR analyzed, taking in consideration that almost half of employees are in the level of 2 to 4 CFR. Conclusion: It was verified an increased percentage of CFR over the university employees. Among the analyzed variables it is possible to verify that the over-weight, TC, low levels of HDL-C and high of LDL-C were the most prevalent CFR.

Keywords: Epidemiology. Public health. Chronic disease. Cardiovascular system.

\section{Introdução}

As doenças cardiovasculares (DCV) têm sido apontadas atualmente como a principal causa de mortalidade e morbidade no Brasil e no mundo (1), assumindo assim o status de epidemia global. Dados de 2002 da Organização Mundial de Saúde (OMS) apontaram que no mundo, do total de 16,7 milhões de óbitos, 7,2 milhões ocorreram por doença arterial coronariana (DAC) (1). No Brasil, atualmente as DCV respondem por cerca de $30 \%$ dos óbitos registrados (2).

Além disso, esse conjunto de doenças crônico-degenerativas é a principal causa de internações hospitalares no setor público pela população adulta, respondendo por $16,22 \%$ do gasto total do Sistema
Único de Saúde (SUS) como a maior causa de gastos em assistência médica (3). No Brasil, em 2005, houve 196.474 internações e 84.945 óbitos registrados no Sistema de Informação de Mortalidade por DAC (4).

Alguns fatores de risco que aumentam a probabilidade de o indivíduo apresentar a DAC são: idade avançada, sexo masculino, fumo, hipertensão arterial, diabetes mellitus, obesidade, sedentarismo, histórico familiar de doença cardiovascular e perfil lipídico alterado (1). Os fatores de risco podem ser encontrados de forma isolada ou associados entre si quando realizada a avaliação de grupos populacionais $(5,6,7)$.

É comum, no ambiente laboral, a constatação de elevada prevalência de fatores de risco, uma vez que estes, além de dependerem das características 
genéticas e ambientais, sofrem influências dos hábitos de vida como alimentação e nível de atividade física habitual. São exemplos os trabalhos com servidores da saúde (5) e funcionários de uma indústria de refrigerantes (6) em que se observou elevada prevalência de fatores de risco nessas populações.

No ambiente universitário, estudos com este estrato da população foram realizados por Moreira, Oliveira, Andrade, Amorim, Oliveira e Doimo (8) e Oliveira, Moreira, Andrade, Amorim, Costa e Marins, (9), em professores universitários; e por Coelho, Caetano, Liberatore, Cordeiro e Souza (10) e Fisberg, Stella, Morimoto, Pasquali, Philippi, Latorre (11) em estudantes universitários. Contudo, trabalhos com servidores públicos federais de ambiente universitário não são habitualmente encontrados, de forma que não está bem estabelecida a prevalência de fatores de risco coronariano nesse grupo.

Estudar a prevalência de fatores de risco em uma determinada categoria profissional, como a de servidores universitários, é interessante porque possibilita dimensionar o risco primário. Essa investigação pode contribuir para a redução da taxa de incidência e prevalência de DAC e fornecer subsídios ao sistema público de saúde e às empresas privadas para a implantação de medidas eficazes de promoção de saúde (12), tendo como meta a diminuição ou eliminação da morbimortalidade por DCV.

Sendo assim, o presente estudo teve como objetivo verificar a prevalência dos fatores de risco cardiovascular em servidores universitários, utilizando as variáveis bioquímicas, antropométricas e pressóricas.

\section{Materiais e métodos}

Participaram do estudo 107 servidores técnico-administrativos da Universidade Federal de Viçosa (UFV), Câmpus Viçosa (MG), todos voluntários, com faixa etária entre 26 e 66 anos. Realizou-se um estudo observacional com delineamento transversal após aprovação no Comitê de Ética em Pesquisa com Seres Humanos da UFV (Of. Ref. n. 020/2010/Comitê de Ética, seguindo a Resolução n. 196/96, que regulamenta sobre estudos com seres humanos).

Inicialmente foi feita uma campanha informativa junto aos servidores, a partir de divulgação no sistema de televisão local, informações no site da universidade e no correio eletrônico pessoal. Aos interessados era explicado todo o procedimento e objetivos da coleta de dados, além de ser marcado um horário individualizado para realização da mesma. Todos os voluntários assinaram o Termo de Consentimento Livre e Esclarecido antes do inicio da coleta.

As avaliações foram realizadas no Laboratório de Performance Humana do Departamento de Educação Física da UFV. As variáveis analisadas foram: massa corporal, estatura, relação cintura-quadril (RCQ), pressão arterial sistólica (PAS) e diastólica (PAD) de repouso. Também foram analisados os níveis séricos de colesterol total (CT), triglicerídeos (TG), lipoproteína de alta densidade (HDL-C) e lipoproteína de baixa densidade (LDL-C). Todos os dados foram armazenados no programa de avaliação física Avaesporte ${ }^{\circledR}$, para que os avaliados tivessem acesso aos seus resultados.

A massa corporal dos indivíduos foi aferida em balança da marca Filizola ${ }^{\circledR}$, modelo ID-M150/4, com precisão de 100 gramas; a estatura foi aferida com um estadiômetro de parede marca Sanny ${ }^{\circledR}$, com precisão de 1 milímetro. 0 índice de massa corporal (IMC) foi calculado com as medidas de massa corporal e estatura. Os pontos de corte de IMC adotados foram os preconizados pela OMS (13). A RCQ foi calculada a partir da divisão do valor obtido na circunferência de cintura pela da circunferência de quadril, medidas estas obtidas com o uso de fita antropométrica metálica de 2 metros da marca Sanny medical ${ }^{\circledR}$. Os pontos de corte adotados foram os preconizados pela OMS (14). Todas as medidas antropométricas utilizadas seguiram as recomendações do International Society for the Advancement of Kinanthropometry (15).

Para a mensuração da pressão arterial, foi utilizado o método auscultatório com auxílio de esfigmomanômetro aneroide marca Unilec ${ }^{\circledR}$, após o avaliado manter repouso na posição sentada por 5 minutos. A classificação dos resultados encontrados foi realizada segundo critérios da Sociedade Brasileira de Cardiologia (16).

Os exames sanguíneos foram realizados por venipunção da artéria cubital em laboratório de análises clínicas. Os resultados para CT, TG, HDL-C e LDL-C foram classificados segundo o Departamento de Aterosclerose da Sociedade Brasileira de Cardiologia (17).

Todos os dados obtidos foram analisados pelo programa estatístico Sigma Stat for Windows, versão 2.03. 0 teste de Komolgorov-Smirnov foi utilizado para verificar a pressuposição de normalidade em todas as variáveis. A análise dos dados consistiu na análise descritiva das variáveis estudadas e no cálculo das prevalências. Foi utilizado o teste $t$ de Student 
não pareado para comparação entre gêneros. A correlação entre as variáveis estudadas foi feita através do coeficiente de correlação de Pearson. Para todos os tratamentos estatísticos adotou-se um nível de significância de $\mathrm{p}<0,05$.

\section{Resultados}

Participaram do estudo 107 avaliados, sendo 64 homens $(59,8 \%)$ e 43 mulheres $(40,2 \%)$, com idade média de $46,11 \pm 10,47$ anos. A Tabela 1 apresenta a análise descritiva das variáveis estudadas.

A prevalência dos fatores de risco cardiovascular (FRC) dos servidores está apresentada no Gráfico 1. Foram encontrados $45 \%$ de excesso de peso (IMC $\geq$ $25,0 \mathrm{~kg} / \mathrm{m}^{2}$ ), sendo que $6,5 \%$ estavam na faixa que classificada como obesidade $\left(\geq 30,0 \mathrm{~kg} / \mathrm{m}^{2}\right)$. Com relação à gordura na região abdominal, $20 \%$ da população apresentou RCQ elevada ( $\geq 0,85$ para as mulheres e $\geq 0,95$ para os homens).

A pressão arterial se apresentou alta (PAS $\geq 140$ mmHg e/ou PAD $\geq 90 \mathrm{mmHg}$ ) em $24 \%$ dos avaliados; $32,8 \%$ dos homens e $11,6 \%$ das mulheres apresentaram valores alterados no momento da avaliação. Em relação à PAS, 9,3\% estavam na faixa de hipertensão estágio 1, não sendo encontrado nenhum caso de hipertensão estágio 2 e 3 . Ao considerar a PAD, 20,6\% estavam na faixa de hipertensão estágio 1 e 2,8\% na faixa de hipertensão estágio 2 .

Com relação aos parâmetros bioquímicos, o colesterol demonstrou a maior elevação com 49\%, sendo encontrado $11,2 \%$ de valores maiores que $240 \mathrm{mg} / \mathrm{dL}$. Quanto aos triglicerídeos, 23\% dos indivíduos apresentaram valores alterados, com 12,1\% de valores classificados como alto (201-499 mg/dL). Em relação ao LDL-C, foram encontrados 31\% de valores acima de $129 \mathrm{mg} / \mathrm{dL}$, com 9,3\% de valores alto ou muito alto ( $>159 \mathrm{mg} / \mathrm{dL}$ ). Foram encontrados valores reduzidos de HDL-C ( $<40 \mathrm{mg} / \mathrm{dL}$ ) em $30 \%$ dos avaliados, envolvendo $37,5 \%$ dos homens e $18,6 \%$ das mulheres (Gráfico 1).

Observou-se que apenas 18,6\% dos avaliados não apresentaram nenhum dos FRC analisados, e aproximadamente a metade dos servidores se encontrou na faixa de 2 a 4 FRC. Entre as mulheres, foram encontrados valores até $4 \mathrm{FRC}$, enquanto entre os homens os FRC ficaram concentrados entre nenhum e 7; entre estes, chamou a atenção um caso em que estavam presentes todas as variáveis analisadas (Gráfico 2).

A Tabela 2 apresenta as correlações entre as variáveis bioquímicas, antropométricas e pressóricas.

Tabela 1 - Caracterização da amostra, segundo o gênero

\begin{tabular}{|c|c|c|c|c|}
\hline \multirow[b]{2}{*}{ Variável } & \multicolumn{2}{|c|}{ Masculino $(n=64)$} & \multicolumn{2}{|c|}{ Feminino $(n=43)$} \\
\hline & média & d.p. & média & d.p. \\
\hline Idade (anos) & 46,59 & 10,57 & 45,39 & 10,39 \\
\hline Peso (kg) & 76,39 & 10,93 & $61,43^{+}$ & 10,12 \\
\hline Estatura (m) & 1,73 & 0,07 & $1,61^{\dagger}$ & 0,06 \\
\hline IMC (kg/m²) & 25,35 & 2,67 & $23,59^{\dagger}$ & 3,36 \\
\hline RCQ & 0,90 & 0,06 & $0,78^{\dagger}$ & 0,07 \\
\hline PAS (mmHg) & 123,98 & 9,64 & $112,79^{+}$ & 8,26 \\
\hline PAD (mmHg) & 82,97 & 6,83 & $76,39^{+}$ & 7,82 \\
\hline CT (mg/dL) & 199,0 & 41,54 & 196,24 & 38,09 \\
\hline $\mathrm{TG}(\mathrm{mg} / \mathrm{dL})$ & 131,36 & 68,90 & 107,78 & 61,23 \\
\hline HDL-C (mg/dL) & 40,95 & 9,68 & $56,03^{\dagger}$ & 12,26 \\
\hline LDL-C (mg/dL) & 132,30 & 38,17 & $114,06^{*}$ & 38,95 \\
\hline
\end{tabular}

Legenda: ${ }^{*}=\mathrm{p}<0,05$ (teste $\mathrm{t}$ ); ${ }^{\dagger}=\mathrm{p}<0,01$ (teste t).

Fonte: Dados da pesquisa. 


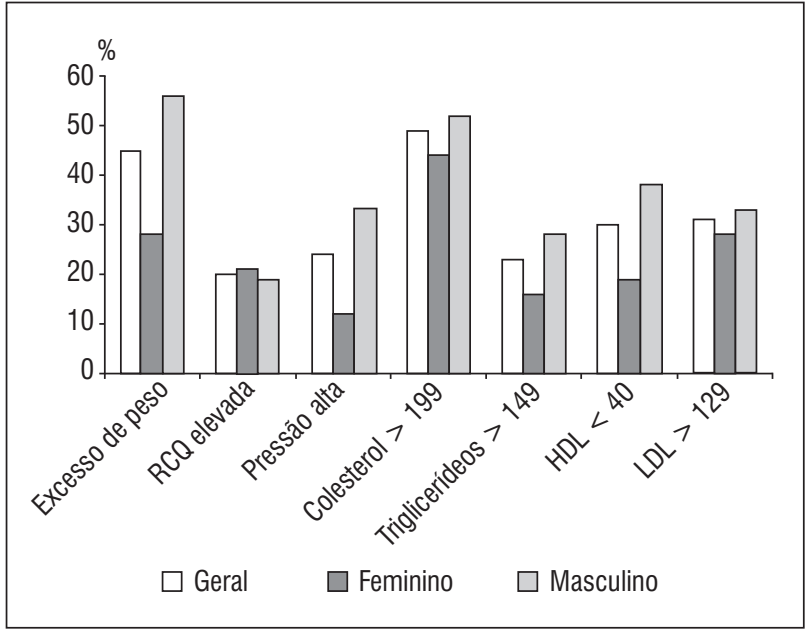

Gráfico 1 - Prevalência dos fatores de risco cardiovascular nos servidores públicos

Fonte: Dados da pesquisa.

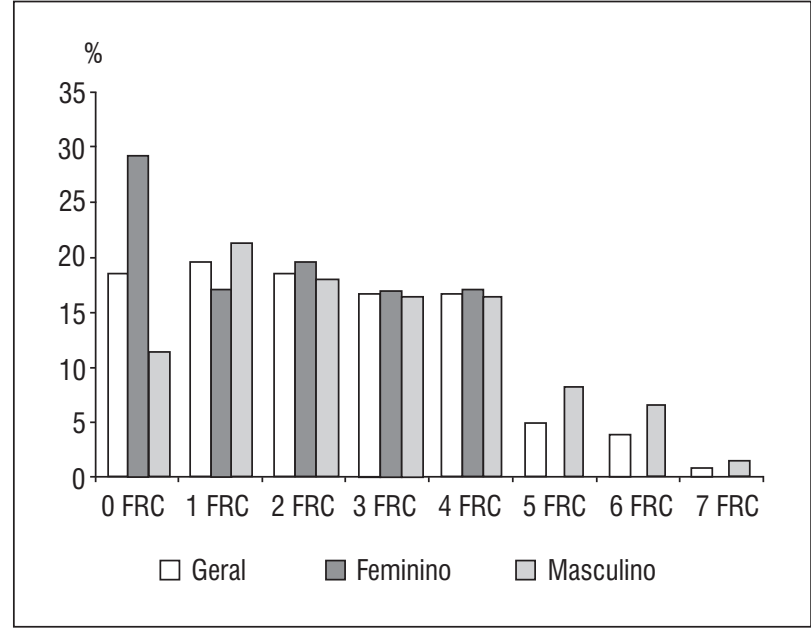

Gráfico 2 - Distribuição dos avaliados em função do número de fatores de risco encontrados

Fonte: Dados da pesquisa.

Tabela 2 - Correlação entre as variáveis analisadas

\begin{tabular}{lccccccc}
\hline Variáveis & IMC & RCQ & PAS & PAD & CT & TG & HDL-C \\
\hline IMC & - & & & & & & \\
RCQ & $0,60^{\dagger}$ & - & & & & & \\
PAS & $0,47^{\dagger}$ & $0,51^{\dagger}$ & - & & & & \\
PAD & $0,47^{\dagger}$ & $0,45^{\dagger}$ & $0,69^{\dagger}$ & - & & & \\
CT & $0,21^{*}$ & 0,18 & 0,09 & 0,18 & - & - & \\
TG & 0,11 & $0,26^{\dagger}$ & $0,27^{\dagger}$ & $0,20^{*}$ & $0,32^{\dagger}$ & - & \\
HDL-C & $-0,12$ & $-0,35^{\dagger}$ & $-0,30^{\dagger}$ & $-0,17$ & 0,16 & $-0,33^{\dagger}$ & - \\
LDL-C & $0,24^{*}$ & $0,29^{\dagger}$ & 0,18 & $0,30^{\dagger}$ & $0,85^{\dagger}$ & 0,07 & $-0,09$ \\
\hline
\end{tabular}

Legenda: ${ }^{*}=p<0,05$ no teste de correlação de Pearson; ${ }^{\dagger}=p<0,01$, no teste de correlação de Pearson.

Fonte: Dados da pesquisa.

\section{Discussão}

Dentre as variáveis antropométricas, foram encontrados $45 \%$ de excesso de peso (IMC $\geq 25,0 \mathrm{~kg} / \mathrm{m}^{2}$ ) na população. Em relação ao gênero, $56,2 \%$ dos indivíduos do sexo masculino e $27,9 \%$ dos avaliados do sexo feminino apresentaram excesso de peso. Em estudo anterior (9) realizado em professores desta mesma universidade, foram encontrados resultados semelhantes entre os homens, com $57,3 \%$ de excesso de peso; porém entre as mulheres os resultados do presente estudo foram inferiores, uma vez que 35,7\% das professoras apresentaram excesso de peso.

Os resultados do presente estudo também se mostraram menores do que os encontrados em servidores da saúde (5); funcionários de indústria de refrigerantes (6); e servidores da Universidade de Brasília (18); porém, foram encontrados resultados mais elevados do que em estudo com funcionários de indústria metalúrgica e siderúrgica (19); e funcionários públicos da cidade de São Paulo (20).

Sabe-se que a obesidade contribui para o aparecimento de inúmeros $\operatorname{FRC}(21,22)$ Nesse sentido, é necessário ressaltar a implantação de medidas preventivas desse fator de risco, para que os quadros de sobrepeso não venham a se tornar obesidade, e consequentemente ocasionar o surgimento de outros FRC na população estudada. Uma série de fatores pode contribuir para o aparecimento de um quadro de sobrepeso, contudo a falta de atividade física e 
uma nutrição não balanceada são determinantes nesse processo, devendo assim fazer parte de ações de intervenção.

O RCQ representa um indicador antropométrico que sinaliza o risco coronariano. Entre os servidores da UFV (Gráfico 1), esse parâmetro se apresentou menos preocupante quando comparados aos indicadores de IMC, pois $20,9 \%$ das mulheres e $28,8 \%$ dos homens estariam em condição de risco coronariano aumentado. Em relação aos valores médios, os resultados desse estrato populacional foram semelhantes aos encontrados em professores da UFV (9). Entretanto, os resultados foram superiores quando comparados com estudo realizado em praticantes de academia na cidade de Florianópolis (SC) (23), e inferiores aos encontrados em população de Salvador (BA) (24).

É importante ressaltar que, em comparação com a obesidade periférica, a obesidade abdominal está associada a uma maior mortalidade (21). Além disso, a obesidade abdominal a faz parte da síndrome metabólica, caracterizada pelo acúmulo de mais de dois fatores de risco em uma mesma pessoa (25), fato que reforça a necessidade de prevenção desse fator de risco na população.

A pressão arterial estava elevada (PAS $\geq 140$ $\mathrm{mmHg}$ e PAD $\geq 90 \mathrm{mmHg}$ ) em $24 \%$ dos avaliados. Quando comparado a dados nacionais, o resultado geral se assemelha aos observados nas cidades de Belo Horizonte, Campo Grande, Maceió, Recife e Vitória. Os homens do presente estudo apresentaram uma prevalência de hipertensão maior do que as mulheres, sendo esse comportamento ainda superior ao observado nas cidades mencionadas (26).

Os valores de pressão arterial também se mostraram maiores do que estudo anterior realizado com professores desta universidade, em que encontraram valores de 16,5\% de hipertensão, com 20,4\% entre os homens e 7,1\% entre as mulheres (8). Além disso, entre a população de professores não foram encontrados casos de hipertensão estágio 2 , conforme observado no presente estudo.

Apesar de não terem sido analisados os fatores escolaridade e renda no presente estudo, esses fatores poderiam explicar as diferenças observadas nas populações de professores e servidores técnico-administrativos da instituição. Isso porque, conforme observado em estudos de Lessa, Araújo, Magalhães, Almeida Filho, Aquino e Costa (27) e Pereira Barreto e Passos (28), quanto maior o nível de escolaridade e renda, menor a prevalência de FRC na população.
É importante ressaltar que a hipertensão arterial é um importante FRC porque causa danos nas artérias, modificando-as. Por ser um fator de risco modificável, é importante enfatizar medidas preventivas como uma alimentação com baixo teor de sódio e atividade física regular, possibilitando obter níveis normais de pressão arterial.

Dentre as variáveis bioquímicas, o CT apresentou a maior elevação ( $49 \%$ de valores $>200 \mathrm{mg} / \mathrm{dL}$ ). 0 segundo parâmetro que apresentou mais indivíduos com níveis inadequados foi o LDL-C, seguido pelo HDL-C e TRI. Em todas as variáveis analisadas o sexo masculino apresentou um pior desempenho em comparação com as mulheres (Figura 1). Em estudos epidemiológicos $(5,10,29,30)$ é possível observar esse comportamento nos parâmetros bioquímicos analisados. 0 fato de os homens apresentarem um pior desempenho torna necessária uma ação mais aguda sobre essa população.

Esses resultados se assemelham aos encontrados em um estudo com servidores da saúde (5) e funcionários de indústria metalúrgica e siderúrgica (19), em que a maioria dos parâmetros de colesterolemia analisados também se encontravam alterados nessas populações. Contudo, os valores do presente estudo se mostraram superiores aos encontrados em funcionários de indústria de refrigerantes (6) e moradores do município de Luzerda (SC) (29).

Os parâmetros bioquímicos analisados demonstram um elevado risco na população com um desequilíbrio colesterolêmico, pois o colesterol elevado contribui para o desenvolvimento da arteriosclerose $(17,22)$, pelo acúmulo de gordura no interior das artérias, associado ainda a um desequilíbrio do LDL-C. É interessante observar a alta prevalência de indivíduos com reduzido HDL-C, pois se sabe que este é responsável pelo transporte reverso do LDL-C, impedindo seu acúmulo nas artérias, sendo um importante protetor cardiovascular. Assim que, o comportamento observado de LDL-C como de HDL-C estão perigosamente aumentados na população avaliada, indicando indiretamente que a nutrição está inadequada.

Em relação ao número de fatores de risco encontrados nos servidores, verificamos que apenas 18,6\% não apresentaram nenhum dos fatores de risco (Figura 2). Isso indica que a maior parte dos servidores apresenta ao menos um FRC. Ao analisar o fator gênero nos homens, quase metade da população se encontrou na faixa de 3 a 7 FRC, enquanto as 
mulheres tiveram até $4 \mathrm{FRC}$ reunidos. Esse resultado se mostra superior ao encontrado em moradores do município de Luzerda (SC), onde mais da metade dos avaliados do estudo não apresentaram nenhum dos fatores de risco analisados (29); entre os homens, inclusive, apenas $11 \%$ apresentaram de 3 a 5 FRC, não sendo encontrado nenhum caso com 6 FRC.

Chama a atenção o elevado número de fatores de risco nos servidores, uma vez que esses podem, com o envelhecimento, aumentar potencialmente o risco de desenvolver problemas cardiovasculares, visto que se trata de uma população com média de idade de 46,11 $\pm 10,47$ anos. Entre o sexo masculino, a prevalência de FRC se apresentou maior, o que merece destaque, pois estes já possuem a questão do gênero como fator de risco não modificável; essa população necessita, portanto, de uma intervenção urgente para redução dos fatores de risco modificáveis.

Quando analisada a relação entre as variáveis estudadas, os níveis pressóricos se relacionaram a TRI, HDL-C e LDL-C; entre as variáveis antropométricas, houve correlação estatisticamente significante entre o IMC e a pressão arterial, CT e LDL-C, sendo que a RCQ se correlacionou com a PAS, PAD, TRI, HDL-C e LDL-C (Tabela 2).

Dentre os parâmetros antropométricos, os resultados encontrados corroboram com a literatura; sabe-se que o sobrepeso e a obesidade, principalmente abdominal, auxiliam para o surgimento de fatores de risco como a hipertensão arterial e as dislipidemias $(21,22,30,31)$. Isso merece destaque, pois segundo o Ministério da Saúde (32) o tratamento da obesidade auxilia na redução da glicemia, da pressão arterial e melhora os níveis lipídicos.

Alguns parâmetros se correlacionaram entre si, como: IMC e RCQ; PAS e PAD; CT, TRI, HDL-C e LDL-C. Observe-se que níveis elevados de sobrepeso e obesidade geral também ocasionam níveis mais altos de gordura localizada na região abdominal, ao passo que, entre os parâmetros bioquímicos, níveis mais altos de CT e TRI se associam com aumento do LDL-C e redução do HDL-C, podendo aumentar o risco cardiovascular.

É importante ressaltar que os resultados encontrados necessitam de mais estudos, tendo em vista que somente foram avaliados $5 \%$ da população total de servidores, porém esses dados exploratórios sinalizam uma possível condição crítica de saúde. É interessante avaliar o nível de padrão dietético e de atividade física desses servidores possibilitando assim uma análise mais crítica, já que poderá auxiliar para a intervenção mais eficiente junto aos servidores.

\section{Conclusão}

Foi verificado um elevado percentual de fatores de risco na população de servidores universitários. Dentre as variáveis analisadas, verificamos que o excesso de peso, colesterol total alterado, níveis reduzidos de HDL-C e aumentados de LDL-C foram os FRC mais prevalentes. Quando analisamos o gênero, observamos que os homens apresentam sistematicamente todos os FRC mais elevados em comparação com as mulheres.

Enfatizamos que é necessária a adoção de medidas de controle e prevenção dos FRC encontrados nessa população, tais como campanhas de conscientização de hábitos de vida saudável, como uma alimentação balanceada e prática regular de atividade física visando diminuir esse quadro alarmante.

\section{Referências}

1. Boletim Brasileiro de Avaliação de Tecnologia em Saúde - BRATS. 2008; Ano III(4).

2. Brasil. Ministério da Saúde. Secretaria executiva. Datasus. Informações em Saúde. Morbidade e informações epidemiológicas. 2010a; [acesso 8 fev. 2010]. Disponível em: http//www.datasus.gov.br

3. Kaiser SE. Aspectos epidemiológicos nas doenças coronariana e cerebrovascular. Rev Socerj. 2004;17(1):11-8.

4. Brasil. Ministério da Saúde. Datasus. [acesso 3 abr. 2007]. Disponível em: http://w3.datasus.gov.br/datasus/datasus.php.

5. Barel M, Louzada GCA, Monteiro HL, Amaral SL. Associação dos fatores de risco para doenças cardiovasculares e qualidade de vida entre servidores da saúde. Rev Bras Educ Fís Esporte. 2010;24(2):293303. doi:10.1590/S1807-55092010000200012.

6. Cassani RSL, Nobre F, Pazin A Filho, Schmidth A. Prevalência de fatores de risco cardiovascular em trabalhadores de uma indústria brasileira. Arq Brasil Cardiol. 2009;92(1):16-22. doi:10.1590/ S0066-782X2009000100004. 
7. Freitas SN, Caiaffa WT, César CC, Faria VA, Nascimento RM, Coelho GLLM. Risco Nutricional na População Urbana de Ouro Preto, Sudeste do Brasil: Estudo de Corações de Ouro Preto. Arq Bras Cardiol. 2007;88(2):191-99. doi:10.1590/ S0066-782X2007000200010.

8. Moreira OC, Oliveira RAR, Andrade F Neto, Amorim W, Oliveira CEP, Doimo LA, et al. Associação entre risco cardiovascular e hipertensão arterial em professores universitários. Rev Bras Educ Fís Esporte. 2011; 25(3):395-404.

9. Oliveira RAR, Moreira OC, Andrade F Neto, Amorim W, Costa EG, Marins JCB. Prevalência de sobrepeso e obesidade em professores da Universidade Federal de Viçosa. Fisioter Mov. 2011;24(4):603-12.

10. Coelho VG, Caetano LF, Liberatore LDR Júnior, Cordeiro JA, Souza DRS. Perfil lipidico e fatores de risco para doenças cardiovasculares em estudantes de Medicina. Arq Bras Cardiol. 2005;85(1). doi:10.1590/ S0066- 782X2005001400011.

11. Fisberg RM, Stella RH, Morimoto JM, Pasquali LS, Philippi ST, Latorre MRDO. Perfil lipídico de estudantes de nutrição e a sua associação com fatores de risco para doenças cardiovasculares. Arq Bras Cardiol. 2001;76(2):137-42. PMid:11294137.

12. Landim MBP, Victor EG. Framingham score for public transportation drivers in the City of Teresina, Piauí. Arq Bras Cardiol. 2006;87(3):315-20. doi:10.1590/ S0066-782X2006001600014.

13. World Health Organization - WHO. Preventing and managing the global epidemic of obesity. Report of a WHO Consultation on Obesity. Geneva; 1997.

14. World Health Organization - WHO. Obesity: preventing and managing the global epidemic. Report of a WHO consultation. World Health Organizations Technical Reports Series. Geneva; 2000.

15. International Society for the Advancement of Kinanthropometry - ISAK. International standards for anthropometric assessment. Adelaid: National Library of Australia; 2001.

16. Sociedade Brasileira de Cardiologia. VI Diretrizes Brasileiras de Hipertensão. Arq Bras Cardiol. 2010; 95(1 Suppl.):1-51.
17. Departamento de Aterosclerose da Sociedade Brasileira de Cardiologia - DASBC. IV Diretriz Brasileira sobre Dislipidemias e Prevenção da Aterosclerose. Arq Bras Cardiol. 2007;88(1 Suppl.).

18. Conceição TV, Gomes FA, Tauil PL, Rosa TT. Valores de pressão arterial e suas associações com fatores de risco cardiovasculares em servidores da Universidade de Brasília. Arq Bras Cardiol. 2006;86(1). doi:10.1590/ S0066-782X2006000100005.

19. Martinez MC, Latorre MRDO. Fatores de risco para hipertensão arterial e diabete melito em trabalhadores de empresa metalúrgica e siderúrgica. Arq Brasil Cardiol. 2006;87:471-79. doi:10.1590/ S0066-782X2006001700012.

20. Neumann AIC, Shirassu MM, Fisberg RM. Consumo de alimentos de risco e proteção para doenças cardiovasculares entre funcionários públicos. Rev Nutr. 2006;19(1).

21. Gomes F, Telo DF, Souza HP, Nicolau JC, Halpern A, Serrano CV Jr. Obesidade e doença arterial coronariana: papel da inflamação vascular. Arq Bras Cardiol. 2010;94(2):273-79. doi:10.1590/ S0066-782X2010000200021.

22. Organização Pan-Americana da Saúde - OPAS. Doenças crônico-degenerativas e obesidade: estratégia mundial sobre alimentação saudável, atividade física e saúde. Brasília; 2003.

23. Grossl T, de Lima LRA, Karasiak FC. Relação entre a gordura corporal e indicadores antropométricos em adultos freqüentadores de academia. Motricidade. 2010;6(2):35-45. doi:10.6063/motricidade.6(2):152.

24. Pitanga FJG, Lessa I. Indicadores antropométricos de obesidade como instrumento de triagem para risco coronariano elevado em adultos na cidade de Salvador-Bahia. Arq Brasil Cardiol. 2005;85(1).

25. International Diabetes Federation - IDF. The IDF consensus worldwide definition of the metabolic syndrome. 2006; [acesso 11 jul. 2011]. Disponível em: http:// www.idf.org/webdata/docs/IDF_Meta_def_final.pdf.

26. Brasil. Ministério da Saúde. Vigitel Brasil 2010: Vigilância de fatores de risco e proteção para doenças crônicas por inquérito telefônico. 2010; [acesso 2 maio 2011]. Disponível em: http://portal.saude.gov.br/portal/arquivos/pdf/vigitel_2010_preliminar_web.pdf. 
27. Lessa I, Araújo MJ, Magalhães L, Almeida M Filho, Aquino E, Costa MCR. Simultaneidade de fatores de risco cardiovascular modificáveis na população adulta de Salvador (BA), Brasil. Rev Panam Salud Publica. 2004;16(2). doi:10.1590/ S1020-49892004000800009.

28. Pereira JC, Barreto SM, Passos VMA. Perfil de risco cardiovascular e autoavaliação da saúde no Brasil: estudo de base populacional. Rev Panam Salud Publica. 2009;25(6). doi:10.1590/ S1020-49892009000600004.

29. Nunes JR Filho, Debastiani D, Nunes AD, Peres KG. Prevalência de fatores de risco cardiovascular em adultos de Luzerda, Santa Catarina, 2006. Arq Bras Cardiol. 2007;89(5):319-24. doi:10.1590/ S0066-782X2007001700007.

30. Viebig RF, Valero MP, Araújo F, Yamada AT, Mansur AJ. Perfil de saúde cardiovascular de uma população adulta da região metropolitana de São Paulo. Arq Bras Cardiol. 2006;86(5). doi:10.1590/ S0066-782X2006000500005.
31. Francischi RPP, Pereira LO, Freitas CS, Klopfer ML, Santos RC, Vieira P, et al. Obesidade: atualização sobre sua etiologia, morbidade e tratamento. Rev Nutr. 2000;13(1):17-29. doi:10.1590/ S1415-52732000000100003.

32. Ministério da Saúde. Caderno de atenção básica: Prevenção Clínica de doença cardiovascular, cerebrovascular e renal crônica. Brasília; 2006; [acesso: 20 out. 2011]. Disponível em: http://bvsms.saude.gov. br/bvs/publicacoes/abcad14.pdf.

Recebido: 15/03/2012

Received: 03/15/2012

Aprovado: 18/09/2012

Approved: 09/18/2012 